\title{
Effects of Molecular Weight and Crystallization Temperature on the Morphology Formation in Asymmetric Diblock Copolymers with a Highly Crystalline Block
}

\author{
Awaludin RoHAdi, Ryuji Endo, Satoshi TANimoto, \\ Shintaro SASAKI, and Shuichi NoJIMA ${ }^{\dagger}$ \\ School of Materials Science, Japan Advanced Institute of Science and Technology \\ (JAIST), Tatsunokuchi, Ishikawa 923-1292, Japan
}

(Received February 9, 2000)

\begin{abstract}
The morphology formed in asymmetric poly( $\varepsilon$-caprolactone)-block-polybutadiene (PCL- $b$-PB) copolymers has been investigated by differential scanning calorimetry (DSC), small-angle X-ray scattering (SAXS), and transmission electron microscopy (TEM) as a function of total molecular weight $M_{n}\left(8000 \leq M_{n} \leq 62000\right)$ and crystallization temperature $T_{\mathrm{c}}\left(-20 \leq T_{\mathrm{c}} \leq 45^{\circ} \mathrm{C}\right)$. All the copolymers have a cylindrical or spherical microdomain structure in the melt with the highly crystalline PCL block inside. In PCL- $b$-PB copolymers with $M_{n} \leq 19000$, the SAXS result showed the morphological transition (microdomain structure $\rightarrow$ lamellar morphology) at each $T_{\mathrm{c}}$ by the crystallization of PCL blocks. The repeating distance of the lamellar morphology increased significantly with increasing $T_{\mathrm{c}}$, as usually observed in the crystallization of homopolymers. The PCL crystallinity $\chi_{\mathrm{c}}$ (i.e., the weight fraction of crystallized PCL blocks against total PCL blocks in the system) was $0.58-0.79$, comparable to that of PCL homopolymer. In PCL- $b$-PB copolymers with $M_{n} \geq 44000$, on the other hand, the morphology did not change by the crystallization at any $T_{\mathrm{c}}$, and $\chi_{\mathrm{c}}$ was extremely reduced $\left(\chi_{c}<0.15\right)$. The morphology formed in PCL- $b$-PB with $M_{n}=30000$ was a combination of above two cases; the repeating distance was independent of $T_{\mathrm{c}}$ and equal to that of the microdomain structure but the morphological transition was confirmed by SAXS and TEM. The relationship between the stabilities of the microdomain structure (or $M_{n}$ ) and lamellar morphology (or $T_{\mathrm{c}}$ ) is qualitatively discussed.

KEY WORDS Crystalline-Amorphous Diblock Copolymer / Microdomain Structure / Lamellar Morphology / Molecular Weight / Crystallization Temperature /
\end{abstract}

The morphology formation in crystalline block copolymers is very complicated by an interplay between the microphase separation and crystallization of constituent blocks. ${ }^{1}$ Many studies have been performed so far on the final morphology of such copolymers as a function of crystallization condition and molecular characteristics. ${ }^{2-20}$ It is established from these studies ${ }^{2-8,13-15}$ that the microdomain structure of low molecular weight copolymers is completely destroyed by the crystallization to form a lamellar morphology, an alternating structure consisting of lamellar crystals and amorphous layers, which is theoretically predicted to be favorable for crystalline block copolymers. ${ }^{21,22}$ This morphological transition arises from the competition between two factors, that is, the strong crystallization force overwhelms the low stability of weakly segregated microdomains. There are, however, limited studies on the morphology of high molecular weight copolymers. Register et al. ${ }^{14}$ have recently investigated the morphology of polyethyleneblock-poly(3-methyl-1-butene) (PE-b-PMB) copolymers as a function of molecular weight, where all the copolymers had a cylindrical morphology in the melt. They found that the sufficiently strong segregation could effectively constrain the crystallization in the existing microdomains. An important factor in their study could be the low levels of crystallinity for the PE block $\left(\chi_{\mathrm{c}} \leq\right.$ 0.35 ); for the copolymers containing a highly crystalline block, it is difficult to achieve the high crystallinity within the curved microdomain and eventually the system will retain the amorphous microdomain structure with no crystallization within it.

The crystallization temperature $T_{\mathrm{c}}$ is another factor to control the morphology formed in polymer materials. In crystalline homopolymers, for example, the crystallization rate decelerates considerably with $T_{\mathrm{c}}$ approaching to the melting temperature $T_{\mathrm{m}}$, resulting in the steady increase of the lamellar thickness and crystallinity. ${ }^{23}$ The increase in the repeating distance of the lamellar morphology is also observed in some crystalline block copolymers such as poly( $\varepsilon$-caprolactone)-block-polybutadiene $(\mathrm{PCL}-b-\mathrm{PB})^{5,8}$ and $\mathrm{PE}-b-\mathrm{PMB},{ }^{14,15}$ indicating that the crystallization kinetics at $T_{\mathrm{c}}$ drives the lamellar morphology formed. In addition, a dramatic change in the final morphology with changing $T_{\mathrm{c}}$ has been recently reported for an ABC triblock copolymer. ${ }^{20}$ Thus, $T_{\mathrm{c}}$, as well as molecular weight $M_{n}$, should be the important factor to control the final morphology formed in block copolymers by the interplay between crystallization and microphase separation.

In our recent study, ${ }^{8}$ we examined the $M_{n}$ dependence of the final morphology formed in PCL- $b$-PB copolymers, where the effect of $T_{\mathrm{c}}$ was neglected. In addition, we used copolymers with relatively low $M_{n}$, so that the morphological transition (microdomain structure $\rightarrow$ lamellar morphology) was observed for all the copolymers investigated. In this study, we examine the morphology in a series of PCL- $b$-PB copolymers as a function of $T_{\mathrm{c}}$ and $M_{n}$, where we use the samples with higher $M_{n}$ by expecting that crystallization occurs within the microdomain structure. The PCL block has inherently high levels of

\footnotetext{
${ }^{\dagger}$ To whom correspondence should be addressed (Phone: +81-761-51-1601, Fax: +81-761-51-1149, E-mail: nojima@jaist.ac.jp).
} 
Table I. Characterization of PCL- $b$-PB copolymers used in this study

\begin{tabular}{|c|c|c|c|c|c|c|c|}
\hline \multirow[b]{2}{*}{ Notation } & \multirow[b]{2}{*}{ Total $M_{n}{ }^{\text {a }}$} & \multirow[b]{2}{*}{$M_{w} / M_{n}^{\mathrm{b}}$} & \multirow{2}{*}{$\begin{array}{c}\mathrm{PCL}: \mathrm{PB}^{\mathrm{c}} \\
(\mathrm{vol} \%)\end{array}$} & \multicolumn{3}{|c|}{ Microstructure of PB chain ${ }^{\mathrm{c}} \%$} & \multirow[b]{2}{*}{$T_{\mathrm{m}}{ }^{\mathrm{d}}\left(T_{\mathrm{c}}\right) /^{\circ} \mathrm{C}$} \\
\hline & & & & cis $-1,4$ & $\operatorname{trans}-1,4$ & 1,2-linkage & \\
\hline M08 & 8000 & 1.09 & $19: 81$ & 36 & 57 & 7 & $51(30)$ \\
\hline M11 & 11000 & 1.15 & $19: 81$ & 34 & 49 & 17 & $51(30)$ \\
\hline M19 & 19000 & 1.25 & $16: 84$ & 41 & 51 & 8 & $55(33)$ \\
\hline M30 & 30000 & 1.11 & $11: 89$ & 35 & 59 & 6 & $54(10)$ \\
\hline M44 & 44000 & 1.07 & $10: 90$ & 34 & 60 & 6 & $54(10)$ \\
\hline M62 & 62000 & 1.13 & $13: 87$ & 38 & 55 & 7 & $57(10)$ \\
\hline
\end{tabular}

crystallinity $\left(\chi_{\mathrm{c}} \geq 0.7\right)$, so that the enthalpic penalty should be large when it is amorphous or crystallizes slightly within the curved microdomain. This point is most different from the PE- $b$-PMB copolymers studied by Register et al., ${ }^{14}$ where the PE block has low levels of crystallinity which is attainable by the partial crystallization within the curved microdomain. We observe the microdomain structure and lamellar morphology by small-angle X-ray scattering (SAXS) and transmission electron microscopy (TEM), and $\chi_{\mathrm{c}}$ and $T_{\mathrm{m}}$ are evaluated by differential scanning calorimetry (DSC). From these results, we clarify quantitatively the effect of $T_{\mathrm{c}}$ and $M_{n}$ on the final morphology in crystalline-amorphous diblock copolymers containing highly crystalline blocks.

\section{EXPERIMENTAL}

\section{Materials}

Poly ( $\varepsilon$-caprolactone )-block-polybutadiene(PCL-b-PB) copolymers were synthesized by a successive anionic polymerization under vacuum with $n$-buthyllithium as an initiator. Details of the synthesis and purification of the samples are described elsewhere. ${ }^{24,25}$ The copolymers obtained were characterized by gel permeation chromatography (GPC), vapor pressure osmometry (VPO) or membrane osmometry, nuclear magnetic resonance $\left({ }^{1} \mathrm{H}\right.$ NMR), and differential scanning calorimetry (DSC). The molecular characteristics of PCL- $b$-PB copolymers are summarized in Table I, where the numeral after ' $M$ ' in Notation represents the total $M_{n}$ in $\mathrm{kg} \mathrm{mol}^{-1} . M_{n}$ changes from 8000 to 62000 with the PCL volume fraction $\phi_{\mathrm{PCL}}$ ranging from 0.10 to 0.19 . It was difficult to prepare copolymers with a fixed $\phi_{\mathrm{PCL}}$ because of the back biting of the living PCL end during the polymerization. ${ }^{26}$ The small $\phi_{\text {PCL }}$ makes us expect the spherical or cylindrical microdomain structure (curved microdomain structure) with the highly crystalline PCL block inside for all the copolymers.

\section{DSC Measurements}

A Perkin-Elmer Pyris 1 or DSC-7 was used to evaluate $T_{\mathrm{m}}$ and $\chi_{\mathrm{c}}$ of the crystallized PCL- $b$-PB. The sample was first annealed at ca. $70^{\circ} \mathrm{C}$ for $15 \mathrm{~min}$, quenched with the maximum rate $\left(\mathrm{ca} .200^{\circ} \mathrm{C} \mathrm{min}^{-1}\right)$ into each $T_{\mathrm{c}}\left(-20 \leq T_{\mathrm{c}}\right.$ $\leq 45^{\circ} \mathrm{C}$ ), crystallized for an enough time at $T_{\mathrm{c}}$, and finally heated at a rate of $5^{\circ} \mathrm{C} \mathrm{min}^{-1}$. The time necessary to complete the crystallization changed significantly with $M_{n}$ and $T_{c}$, so that we checked the time dependence of $\chi_{\mathrm{c}}$ to find the end of crystallization. $T_{\mathrm{m}}$ was defined as the maximum temperature of the endothermic peak and $\chi_{\mathrm{c}}$ was calculated by $\chi_{\mathrm{c}}=\Delta H /\left(\Delta H^{*} \phi_{\mathrm{PCL}}^{\prime}\right)$, where $\Delta H$ and
$\Delta H^{*}$ are the heat of fusion of the sample and perfect PCL crystal $\left(=135.44 \mathrm{~J} \mathrm{~g}^{-1}\right)^{27}$ and $\phi_{\mathrm{PCL}}^{\prime}$ is the weight fraction of PCL blocks in the sample. Two endothermic peaks were observed in some cases, for which peak separation was performed by the computational method to evaluate each $T_{\mathrm{m}}$ and $\chi_{\mathrm{c}}$.

\section{SAXS Measurements}

The SAXS measurements were performed with a point focusing optics and a position sensitive proportional counter (PSPC) with an effective length of $10 \mathrm{~cm}$. Details of the optics, instrumentation, and data processing are described elsewhere. ${ }^{12,28,29}$ The SAXS curve after various corrections was finally obtained as a function of wave number $s$ defined as $s=(2 / \lambda) \sin \theta$, where $\lambda$ is the wave length of the incident X-ray $(=0.1542 \mathrm{~nm})$ and $2 \theta$ is the scattering angle.

The copolymers were first annealed at $c a .70^{\circ} \mathrm{C}$ for 15 min, quenched into each $T_{\mathrm{c}}\left(-20 \leq T_{\mathrm{c}} \leq 35^{\circ} \mathrm{C}\right)$, and crystallized at $T_{\mathrm{c}}$ for an enough time until the significant change in the SAXS curve was not observed, where the time dependence of $\chi_{c}$ obtained by DSC measurements was also used to judge the end of crystallization.

\section{TEM Observations}

The morphology after crystallization of PCL blocks was observed by TEM, together with the microdomain structure in the melt. The sample crystallized at each $T_{\mathrm{c}}$ was first trimmed at $-140^{\circ} \mathrm{C}$ with a glass knife followed by stained with $\mathrm{O}_{s} \mathrm{O}_{4}$ vapor for $24 \mathrm{~h}$, and finally microtomed with a diamond knife into a thin film with a thickness of $c a .50 \mathrm{~nm}$. The PB block existing in the system was preferentially stained by $\mathrm{O}_{s} \mathrm{O}_{4}$. TEM images were obtained with a Hitachi H-7100 TEM operating at $100 \mathrm{kV}$. The repeating distance of the morphology was roughly evaluated from the TEM image and compared with that obtained from SAXS measurements.

\section{RESULTS}

\section{SAXS Measurements}

The morphology formed by the crystallization of PCL blocks at each $T_{\mathrm{c}}$, as well as the microdomain structure in the melt, was investigated by SAXS and the typical results are plotted in Figure 1. The SAXS curve of M08 (and also M11 and M19) changes dramatically by the crystallization $\left(70^{\circ} \mathrm{C} \rightarrow-10-35^{\circ} \mathrm{C}\right.$ in Figure 1a), indicating that the morphological transition occurs in the system. The SAXS curve of the crystallized M08 has a faint second peak at $2 s^{*}$, where $s^{*}$ is the position of the first-order peak, suggesting that the (cylindrical or 

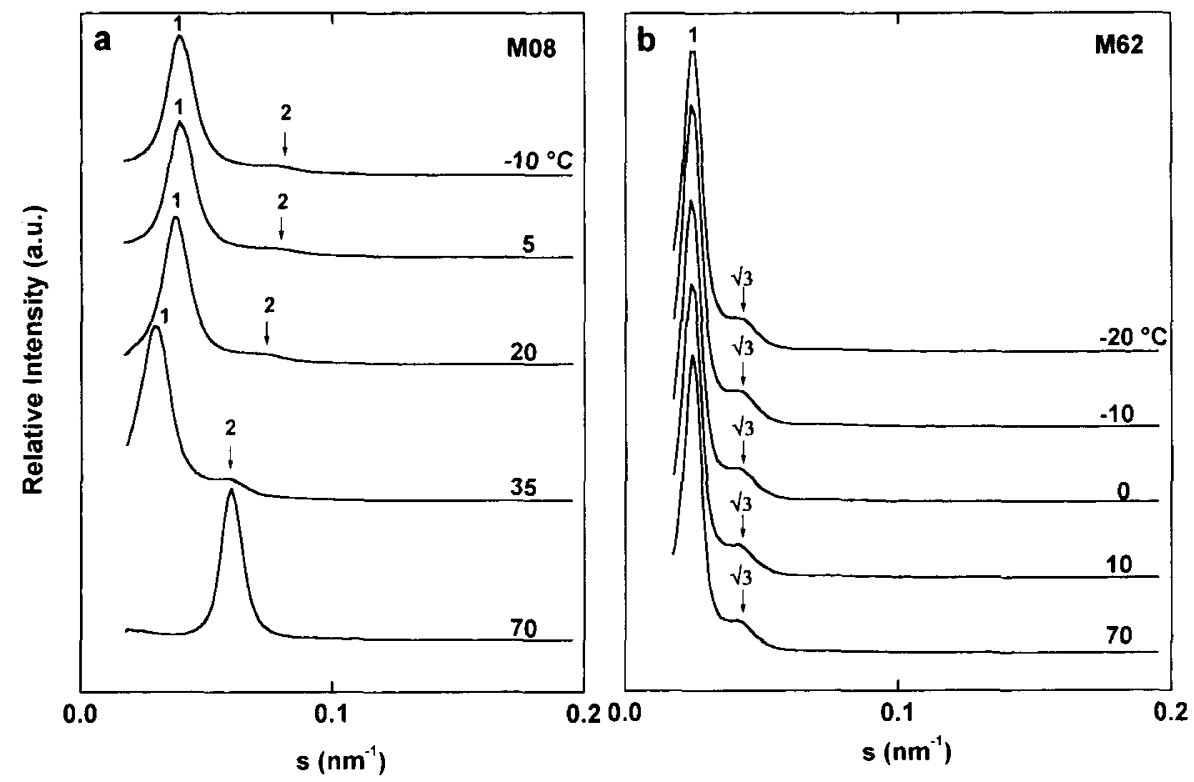

Figure 1. SAXS intensity plotted against $s(=(2 / \lambda) \sin \theta, 2 \theta$ is the scattering angle and $\lambda$ is the X-ray wave length ( $=0.1542 \mathrm{~nm}))$ for $\mathrm{M}$ 08 (a) and M62 (b) crystallized at various temperatures indicated on each curve. The bottom SAXS curve in each panel was obtained from the microphase-separated samples in the melt. The SAXS curve of M08 at $70^{\circ} \mathrm{C}$ is enlarged by a factor of 2 for clarity.

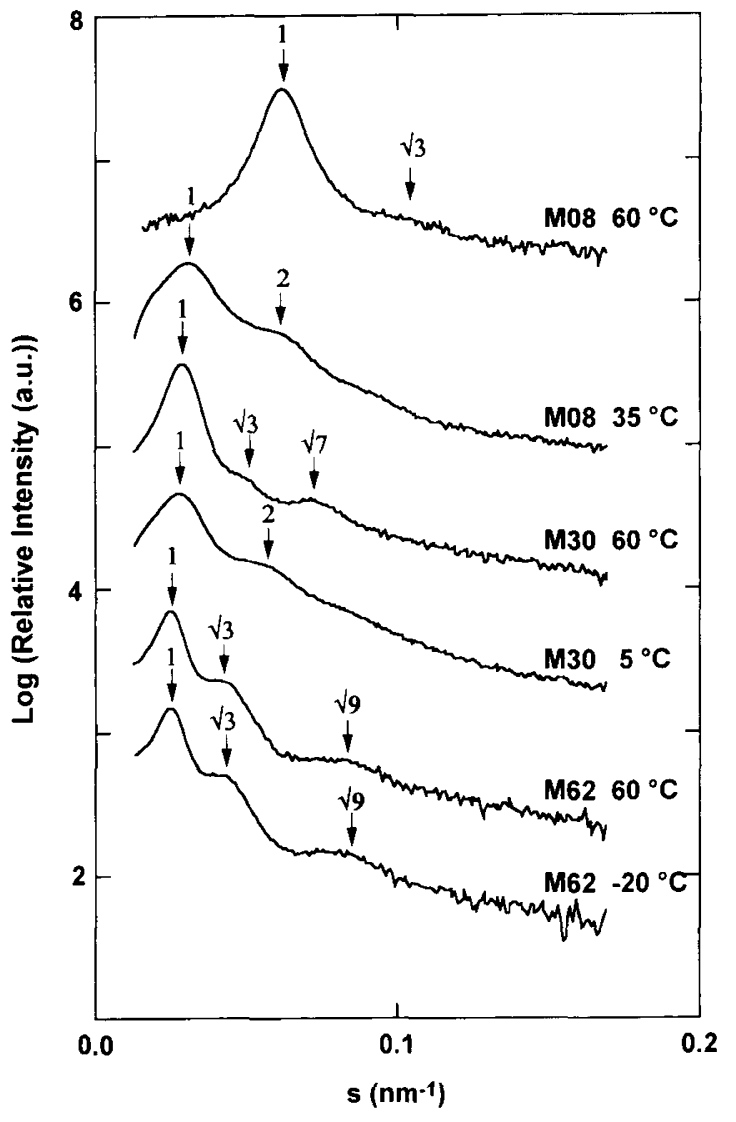

Figure 2. Logarithmical plot of the SAXS curve of M08, M30, and M62 in the melt $\left(60^{\circ} \mathrm{C}\right)$ and after the crystallization of PCL blocks $\left(-20-35^{\circ} \mathrm{C}\right)$.

spherical) microdomain structure is transformed into the lamellar morphology, an alternating structure consisting of lamellar crystals and amorphous layers, even if $\phi_{\mathrm{PCL}}$ is as small as $0.19 .^{6}$

The SAXS curves of M62 are different from those of
M08, where $s^{*}$ (and also the position of the second-order peak $\left(=\sqrt{3} s^{*}\right)$ ) does not change by the crystallization $\left(70^{\circ} \mathrm{C} \rightarrow-20-10^{\circ} \mathrm{C}\right.$ in Figure $\left.1 \mathrm{~b}\right)$. This fact indicates that the microdomain structure is retained even in the temperature range where the PCL chain usually crystallizes rapidly to form the lamellar morphology. The confined space with the curved surface (i.e., the cylindrical or spherical microdomains with the PCL block inside) will restrict the crystallization of PCL blocks considerably. M30 and M44 showed the similar SAXS curves with those of M62 but $\chi_{\mathrm{c}}$ for M 30 was much larger than that for M44 and M62, as described later.

In Figure 2, we plotted the SAXS intensity logarithmically to emphasize the difference in the shape of SAXS curves before and after the crystallization for M08, M30, and M62. We find from Figure 2 that although $s^{*}$ for M30 does not change by the crystallization, the positions of second- and third-order peaks are entirely different between two SAXS curves of M30 $\left(\sqrt{3} s^{*}\right.$ and $\left.\sqrt{7} s^{*} \rightarrow 2 s^{*}\right)$, indicating that the morphological transition (spherical or cylindrical microdomain structure $\rightarrow$ lamellar morphology) has occurred. This is a special case of the morphological transition, where the lamellar morphology forms from the pre-existing microdomain structure with the repeating distance being constant, and therefore the constituent block does not need to diffuse extensively. An epitaxial relationship was recently observed between the microdomain in the melt and lamellar morphology for a selected polyethylene-block-poly(1-ethylethylene $)^{10}$ or poly(oxyethylene)-block-poly(oxybuthylene) ${ }^{13}$ when it was crystallized rapidly from the melt, where the cylindrical microdomain structure turned into the lamellar morphology without changing the principal repeating distance of the morphology. This epitaxy seems to relate intimately to the crystallization kinetics because it appears only by a deep quench, where the crystallization occurs rapidly. It is worth noting that M30 shows this relation for all $T_{\mathrm{c}}$ 's ranging from -20 to $20^{\circ} \mathrm{C}$, 

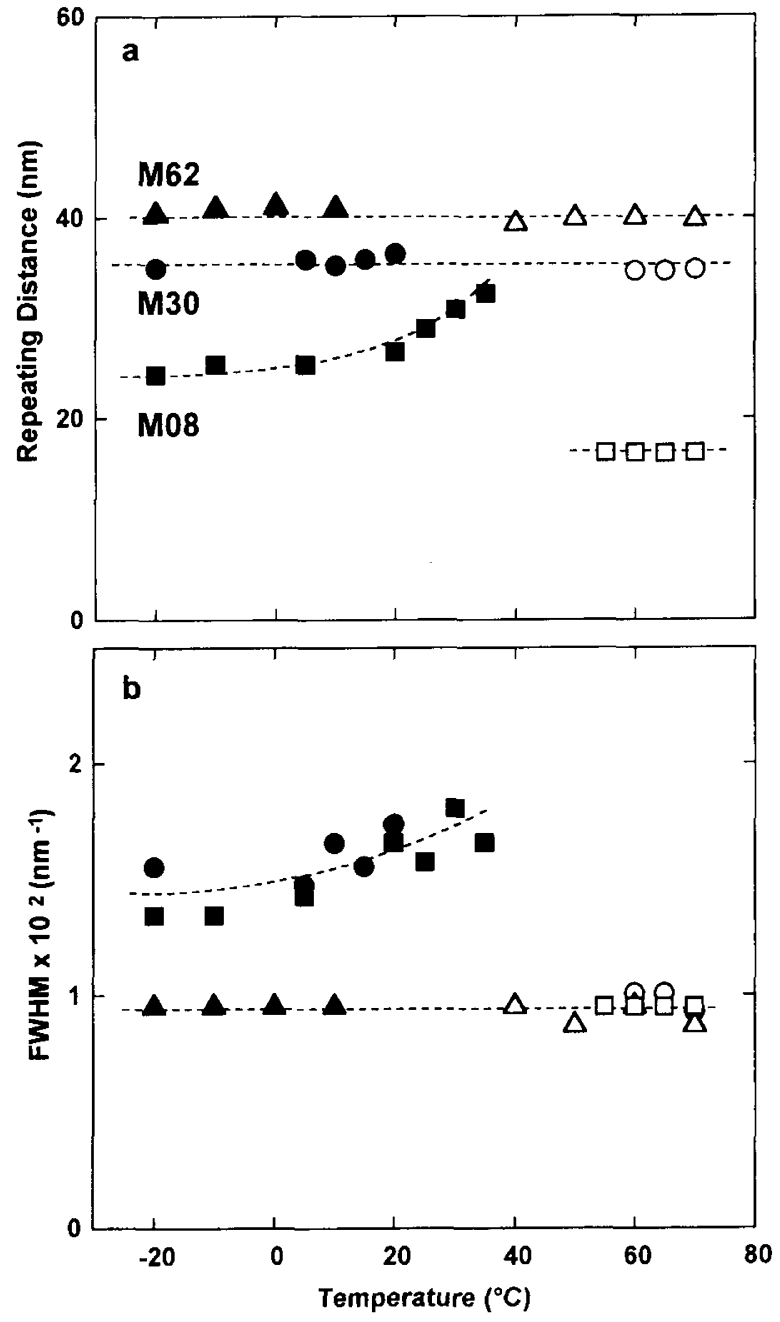

Figure 3. Repeating distance $\left(=1 / s^{*}\right)$ (a) and FWHM of the principal SAXS peak (b) plotted against temperature for M08 $(\square, \boldsymbol{\nabla})$, M30 $(\bigcirc, \boldsymbol{O})$, and M62 $(\triangle, \Delta)$. The closed symbols represent that the crystallization of PCL blocks is detected by the DSC measurement.

suggesting that the present epitaxial transition is not merely controlled by the crystallization kinetics at $T_{\mathrm{c}}$.

Figure 3 summarizes the temperature dependence of the repeating distance $\left(=1 / s^{*}\right)$ (a) and full width at half maximum (FWHM) of the principal intensity peak (b) for M08, M30, and M62. FWHM is a measure of the regularity of the morphology formed in the system; FWHM becomes smaller with increasing the regularity. As mentioned above, we find from Figure $3 a$ that the repeating distance of M30 and M62 (and also M44) is constant irrespective of $T_{\mathrm{c}}$ and equal to that of the microdomain structure, while it increases steadily with increasing $T_{\mathrm{c}}$ for M08 (and also M11 and M19) and is fairly larger than that of the microdomain structure. Figure $3 \mathrm{~b}$ shows the significant difference in the $T_{\mathrm{c}}$ dependence of FWHM among M08, M30, and M62. FWHM of M08 and M30 increases steadily with increasing $T_{\mathrm{c}}$ and larger than that of the microdomain structure. This means that the regularity decreases suddenly by the crystallization and that the lamellar morphology formed is less regular compared to the microdomain structure. FWHM for M62 is, on the other hand, independent of temperature, indicating that the microdomain structure does not deform at
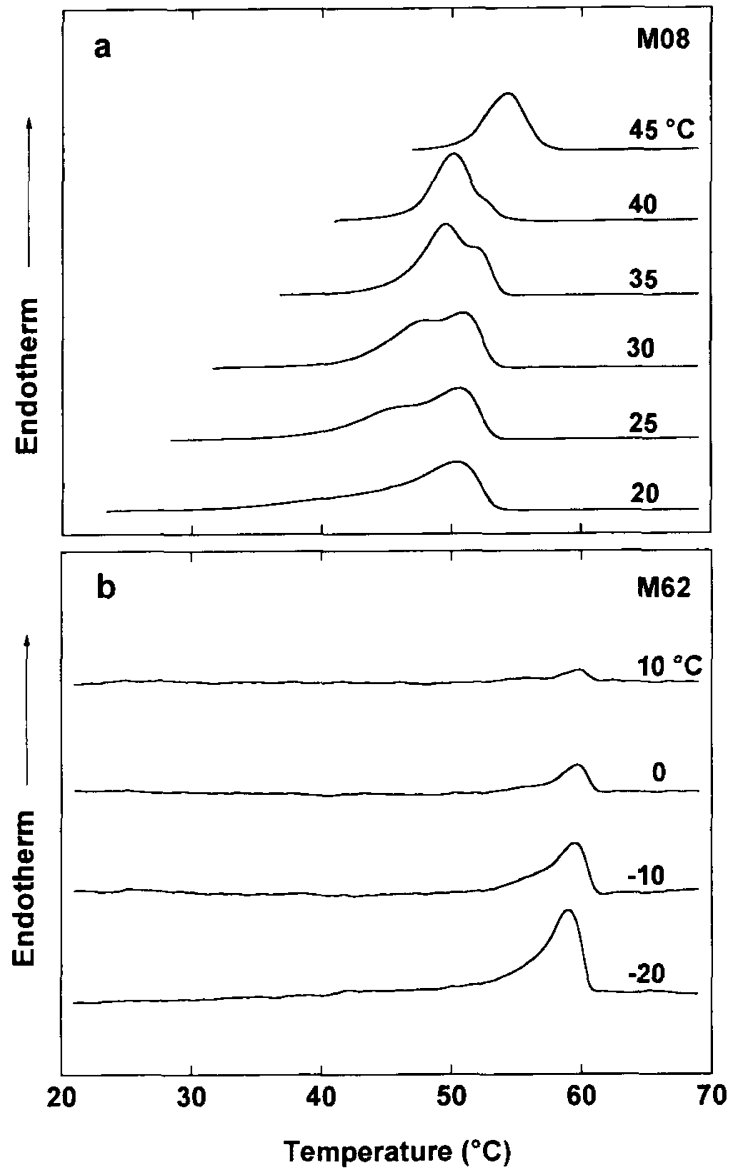

Figure 4. DSC melting curves for M08 (a) and M62 (b) crystallized at various temperatures indicated on each curve. The heating rate is $5^{\circ} \mathrm{C} \min ^{-1}$. The ordinate in $b$ is enlarged by a factor of 3 for clarity.

all even if the PCL block crystallizes partially $\left(\chi_{\mathrm{c}}<0.15\right)$ within the microdomain. A slight deformation of the microdomain structure was previously observed for the crosslinked PCL- $b-\mathrm{PB},{ }^{12}$ where $\chi_{\mathrm{c}}$ was significantly larger $\left(\chi_{\mathrm{c}} \sim 0.25\right)$ than the present case. Therefore, $\chi_{\mathrm{c}}$, which may be changed by molecular characteristics of the constituent block copolymers, affects significantly the regularity of the microdomain structure when the PCL block crystallizes partially.

We find from the SAXS results that the morphological transition primarily depends on $M_{n}$, that is, the stability of the microdomain structure in the melt. The crystallization temperature, on the other hand, affects delicately the final morphology; when $M_{n}$ is low $\left(M_{n} \leq 19000\right) T_{\mathrm{c}}$ controls the crystallization rate exclusively to decide the details of the lamellar morphology formed (lamellar thickness, crystallinity, folded number of PCL blocks, elongation degree of $\mathrm{PB}$ blocks, and so on). When $M_{n}$ is larger than $30000, T_{\mathrm{c}}$ does not provide the visible change in the final morphology when it is observed by SAXS even if the morphological transition takes place (for $M_{n}$ $=30000$ ) or $\operatorname{not}\left(\right.$ for $\left.M_{n} \geq 44000\right)$.

\section{DSC Measurements}

Figure 4 shows the DSC melting curves of M08 and M62 crystallized at each $T_{\mathrm{c}}$ indicated, where the ordinate in Figure $4 \mathrm{~b}$ is enlarged by a factor of 3 . We find 


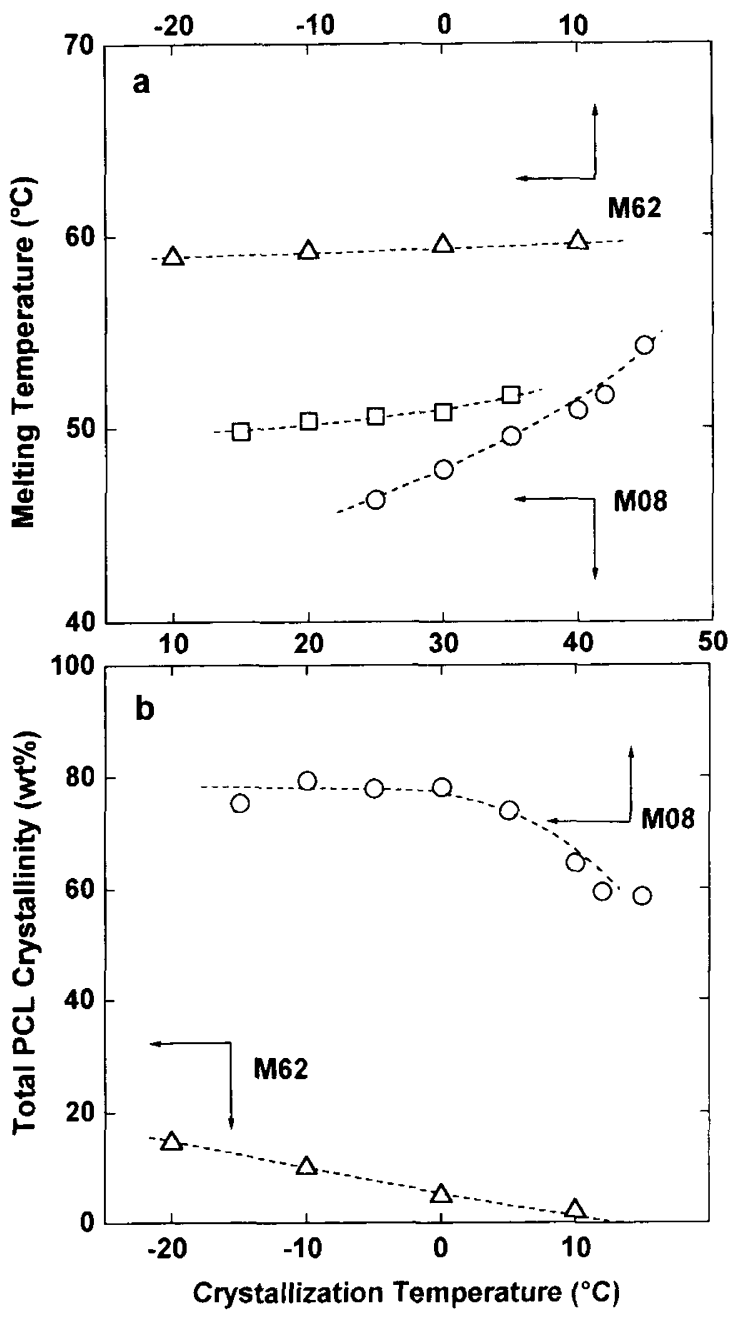

Figure 5. Melting temperature (a) and total PCL crystallinity in wt\% (b) plotted against crystallization temperature for M08 $(\mathrm{O}$ and $\square)$ and $\mathrm{M} 62(\triangle)$.

that the melting behavior of M08 changes significantly with changing $T_{\mathrm{c}}$ and the melting enthalpy (i.e., $\chi_{\mathrm{c}}$ ) is very large. M11, M19, and M30 showed the similar melting behavior with that of M08. The melting endotherm of M62, on the other hand, is very small though $\chi_{\mathrm{c}}$ depends significantly on $T_{\mathrm{c}}$ with the peak position (i.e., $T_{\mathrm{m}}$ ) being unchanged. M44 showed the similar DSC curve with that of M62. Thus, the melting behavior (and therefore the final morphology) of the present PCL- $b$-PB copolymers is classified into two cases, that is, Figures $4 \mathrm{a}$ and $4 \mathrm{~b}$.

The DSC curve of M08 has two endothermic peaks in a limited range of $T_{\mathrm{c}}\left(25 \leq T_{\mathrm{c}} \leq 40^{\circ} \mathrm{C}\right)$ and seems to be represented by a combination of the endothermic curves at $T_{\mathrm{c}}=20$ and $45^{\circ} \mathrm{C}$ after shifting them along the temperature axis. The double melting phenomenon is sometimes observed for the melting of homopolymers and ascribed to the reorganization of the lamellar morphology during heating. ${ }^{30,31}$ Therefore the characteristic DSC curve depends critically on the heating rate; when the heating rate is small there is an enough time for thin lamellae to melt and recrystallize, so that we get clear bimodal DSC curve, while when the heating rate is large, the lamella cannot thicken during heating to form the broad single DSC curve. In the present case, however, the melting be-

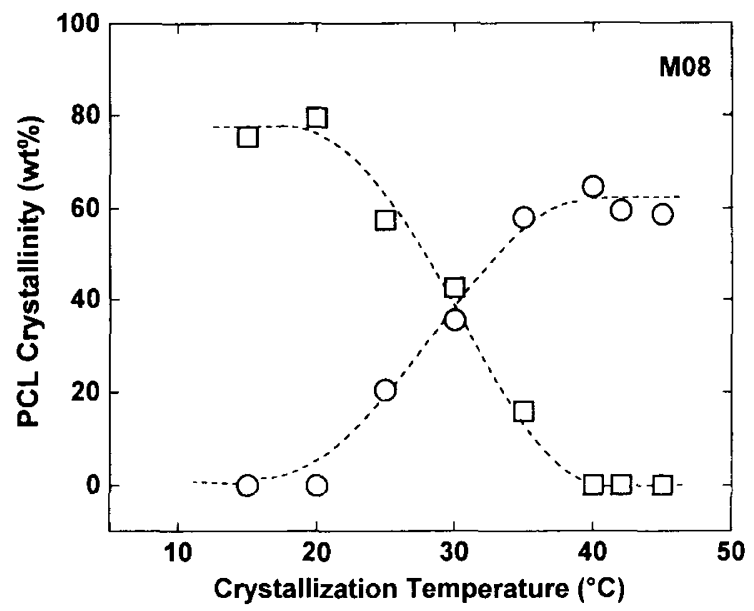

Figure 6. PCL crystallinities in wt\% plotted against crystallization temperature when the bimodal endothermic peak of M08 is divided into two single peaks. $\bigcirc$ : low-temperature peak, $\square$ : hightemperature peak.

havior represented by Figure $4 \mathrm{a}$ did not depend on the heating rate ranging from $3^{\circ} \mathrm{C} \mathrm{min}^{-1}$ to $20^{\circ} \mathrm{C} \mathrm{min}{ }^{-1}$, suggesting that the bimodal DSC curve does not arise from the morphological reorganization during heating. This double melting behavior was previously observed for some block copolymers containing crystalline blocks ${ }^{2,11}$; Gan et al. ${ }^{11}$, for example, observed the similar melting behavior for poly(tetrahydrofuran)-block-poly(ethylene oxide) (PTHF- $b$-PEO) copolymers and concluded that the double melting behavior is not ascribed to the melting and recrystallization of thin lamellae during heating but the existence of two slightly different morphologies formed under the nonequilibrium crystallization at $T_{\mathrm{c}}$. We discuss these morphologies later, together with the results of TEM and SAXS.

Figure 5 shows the plot of $T_{m}$ (a) and $\chi_{c}$ (b) against $T_{c}$ for M08 and M62. As mentioned above, M08 shows two melting peaks in the DSC curve, so that higher and lower melting temperatures are plotted in Figure 5a. The value of $T_{m}$ for M62 is almost independent of $T_{c}$ and close to that of PCL homopolymers, while $\chi_{\mathrm{c}}$ is extremely small $\left(\chi_{\mathrm{c}}<0.15\right)$. In addition, the crystallization could not be confirmed at $T_{\mathrm{c}} \geq 15^{\circ} \mathrm{C}$ within the experimentally accessible time (more than one week). It is surprising that the highly crystalline PCL chain shows such a low crystallinity at $T_{\mathrm{c}} \leq 10^{\circ} \mathrm{C}$ and no crystallinity at $T_{\mathrm{c}} \geq$ $15^{\circ} \mathrm{C}$. Though $\chi_{\mathrm{c}}$ for M08 is large $\left(0.58 \leq \chi_{\mathrm{c}} \leq 0.79\right), T_{\mathrm{m}}$ changes complicatedly with changing $T_{c}$; the lower $(\bigcirc)$ and higher $(\square)$ melting temperatures increase with increasing $T_{\mathrm{c}}$ and merge into one at about $40^{\circ} \mathrm{C}$. This change in $T_{\mathrm{m}}$ against $T_{\mathrm{c}}$ is similar to that reported by Gan et al. for PTHF- $b$-PEO copolymers, ${ }^{11}$ and seems to be a general phenomenon observed in crystalline block copolymers. We notice from Figure $5 \mathrm{~b}$ that $\chi_{\mathrm{c}}$ is constant or decreases slightly with increasing $T_{\mathrm{c}}$. This is not usually observed for the melting of homopolymers, where $\chi_{\mathrm{c}}$ increases steadily with increasing $T_{\mathrm{c}}$ which is attributed to the slower crystallization at higher $T_{\mathrm{c}}$ to make the lamella thicker. Figure 5b suggests that the crystallization mechanism of block copolymers is different from that of homopolymers, that is, the lamellar thickening is not the only factor to reduce the free energy of the system. ${ }^{21,22}$ 

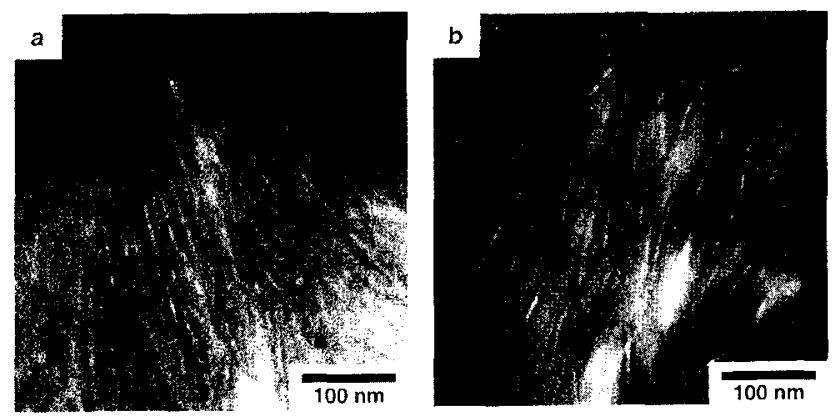

Figure 7. TEM micrographs of M11 crystallized at $10^{\circ} \mathrm{C}$ (a) and $45^{\circ} \mathrm{C}$ (b). The black region is the $\mathrm{PB}$ chains stained by $\mathrm{O}_{s} \mathrm{O}_{4}$.

To understand the bimodal character of the DSC thermogram shown in Figure $4 \mathrm{a}$ in more detail, we divided the DSC curve into two single curves (ca., those of $T_{\mathrm{c}}=$ 20 and $45^{\circ} \mathrm{C}$ ) by the computational method. The $T_{\mathrm{c}}$ dependence of the lower-peak crystallinity $\chi_{1}$ and higherpeak crystallinity $\chi_{\mathrm{h}}$ is shown in Figure 6 , where $\chi_{1}$ increases and simultaneously $\chi_{\mathrm{h}}$ decreases with increasing $T_{\mathrm{c}}$ and both meet at $T_{\mathrm{c}} \sim 30^{\circ} \mathrm{C}$. We expect from Figure 6 that two slightly different morphologies coexist in the system and their fractions change significantly with $T_{\text {c. }}$.

The DSC results reveal that there is a large difference in the melting behavior between PCL- $b$-PB copolymers with $M_{n} \leq 30000$ and $M_{n} \geq 44000$, for which the morphological difference is responsible, as clarified by SAXS; the former copolymers form the lamellar morphology and the latter the microdomain structure in which the PCL block crystallizes slightly (crystallized microdomain structure). Register et al. observed the small difference in the crystallinity of $\mathrm{PE}$ blocks between the lamellar morphology $\left(0.31 \leq \chi_{\mathrm{c}} \leq 0.36\right)$ and crystallized microdomain structure $\left(0.26 \leq \chi_{\mathrm{c}} \leq 0.32\right)$ for a series of PE- $b$-PMB copolymers, ${ }^{14}$ where the crystallinity levels of $\mathrm{PE}$ blocks are not high compared with the present PCL block $\left(0.58 \leq \chi_{\mathrm{c}} \leq 0.79\right)$. When the constituent block is highly crystalline, the system tends to reorganize the structure into the lamellar morphology or to keep the microdomain structure with no crystal inside, since it is impossible to maintain the high crystallinity within the curved microdomain. The system chooses one of the two extreme morphologies depending both on the stability of the microdomains and crystallization force; when the crystallization overwhelms the microdomain stability the lamellar morphology appears, while the microdomain structure is extremely stable it remains without any crystallization inside. This point is most different between the crystalline blocks with low and high levels of crystallinity.

\section{TEM Observations}

From DSC and SAXS measurements, we have two questions about the morphology formed in the present PCL- $b$-PB copolymers; (1) are there two morphologies existing in the crystallized M08, as indicated by the double melting curve (Figure $4 \mathrm{a}$ )? and (2) whether the morphological transition occurs certainly in M30 by the crystallization of PCL blocks. We took TEM pictures to clarify these points.
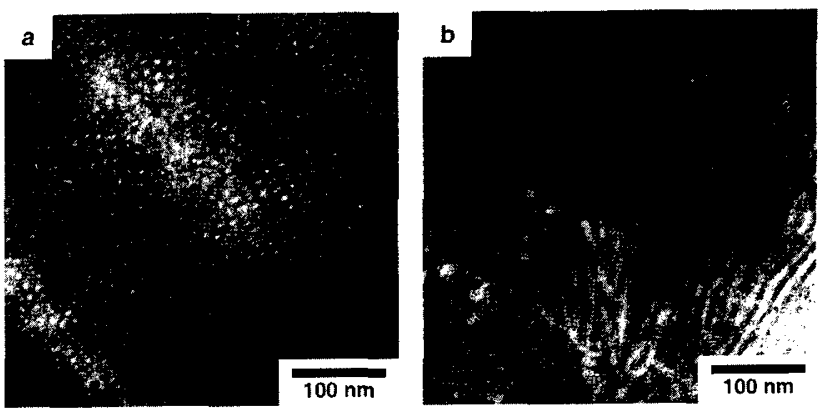

Figure 8. TEM micrographs of M30 in the melt (a) and crystallized at $5^{\circ} \mathrm{C}(\mathrm{b})$.

Figure 7 shows the lamellar morphology formed in M11 crystallized at $T_{\mathrm{c}}=10^{\circ} \mathrm{C}$ (a) and $T_{\mathrm{c}}=45^{\circ} \mathrm{C}(\mathrm{b})$, where we know from Figure 6 that only one of the morphologies is present in the system. (The melting behavior of M11 is similar with that of M08.) Both TEM images show the lamellar morphology with relatively thin PCL lamellae (white strips), which is similar with the TEM pictures reported for crystalline block copolymers. ${ }^{20,32}$ The repeating distance of this lamellar morphology is ca. 18 $\mathrm{nm}$ for a and $c a .29 \mathrm{~nm}$ for $\mathrm{b}$ and agrees reasonably with the SAXS result (not shown here). We cannot find any distinct difference in the morphology between Figures $7 \mathrm{a}$ and $7 \mathrm{~b}$. We infer that even if there is a difference in the lamellar morphology, it might be the internal structure of lamellar crystal or the interfacial structure between lamellae and amorphous layers, which are indistinguishable from Figure 7 with the limited contrast. But we can say that the morphological difference suggested by the double melting curve does not imply the essential difference in the morphology such as the difference between the lamellar morphology and crystallized microdomain structure, but the difference in the minor portion within the lamellar morphology.

Finally, Figure 8 shows the TEM image of M30 before (a) and after (b) crystallization of PCL blocks. We can find the spherical microdomain structure in Figure $8 \mathrm{a}$ and lamellar morphology in Figure $8 \mathrm{~b}$ though the contrast is again very limited. The repeating distance is almost equal for these morphologies (ca., $27 \mathrm{~nm}$ ), as expected from Figure $3 \mathrm{a}$, though it is moderately smaller than that obtained by SAXS ( $\sim 35 \mathrm{~nm})$. Figure 8 indicates evidently that the morphological transition (spherical microdomain structure $\rightarrow$ lamellar morphology) occurs by the crystallization, which is consistent with the SAXS and DSC results shown in Figures 2 and 5.

\section{DISCUSSION}

Here, we consider the relationship intuitively between the stabilities of the microdomain structure in the melt and lamellar morphology formed at $T_{\mathrm{c}}$ to explain the present results qualitatively.

Apart from the complicated effects characteristic of block copolymers, the decrease in free energy (divided by $k T$ ) by the crystallization at $T_{\mathrm{c}}, \Delta F_{\mathrm{c}}$, is mainly composed of (negative) crystal free energy term (which is proportional to the crystal volume) and (positive) surface free energy term (which is proportional to the surface area of 


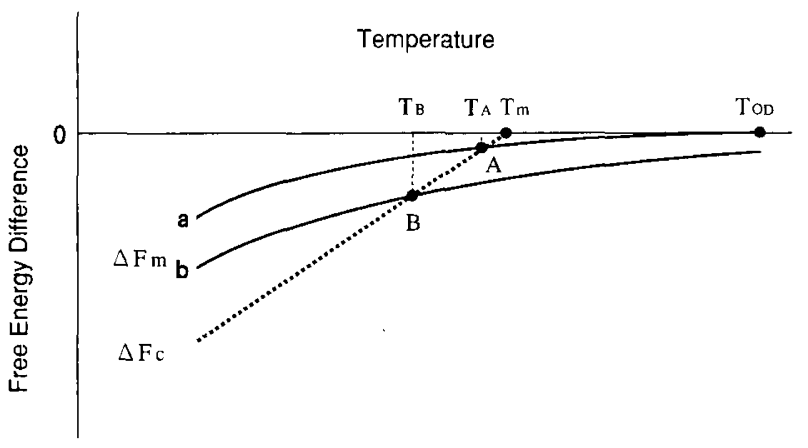

Figure 9. Free energy difference as a function of temperature for the microdomain structure $\left(\Delta F_{\mathrm{m}}\right)$ and lamellar morphology $\left(\Delta F_{\mathrm{c}}\right)$ Curve a represents the case for $T_{\mathrm{OD}}>T_{\mathrm{m}}$ and curve $\mathrm{b}$ for $T_{\mathrm{OD}} \gg T_{\mathrm{m}}$.

the crystal). If we assume that the enthalpic and entropic differences between the crystal and homogeneous melt are temperature-independent ${ }^{23}$ and that $\Delta F_{\mathrm{c}}=0$ at $T_{\mathrm{c}}=T_{\mathrm{m}}$, we simply obtain,

$$
\Delta F_{\mathrm{c}} \propto-C\left(T_{\mathrm{m}}-T_{\mathrm{c}}\right)
$$

where $C$ is a positive constant independent of $T_{\mathrm{c}}$.

The theory to investigate the stability of the microdomain structure formed in amorphous diblock copolymers was recently proposed by using a self-consistent mean field theory, ${ }^{33,34}$ where it covers the temperatures ranging from the weak to the strong segregation regions. The free energy difference between the microdomain structure and the homogeneous melt, $\Delta F_{\mathrm{m}}$, can be expressed approximately as,

$$
\Delta F_{\mathrm{m}} \propto-\frac{\left(\chi M_{n}-\alpha\right)^{2}}{\chi M_{n}^{2}}
$$

where $M_{n}$ is the total molecular weight of the constituent block copolymer, $\chi$ is the Flory-Huggins interaction parameter between the blocks, and $\alpha$ is a constant depending on the type of the microdomain structure and the volume fraction of one of the blocks. By assuming that $\chi$ is inversely proportional to $T$ (i.e., $\chi \propto 1 / T$ ), we get,

$$
\Delta F_{\mathrm{m}} \propto-C^{\prime} \frac{\left(T_{\mathrm{OD}}-T\right)^{2}}{T}
$$

where $C^{\prime}$ is also a positive constant independent of $T$ and $T_{\mathrm{OD}}$ is the order-disorder transition temperature of the microphase separation.

The relation between $\Delta F_{\mathrm{c}}$ and $\Delta F_{\mathrm{m}}$ against temperature is depicted schematically in Figure 9, where curve a represents the case of $T_{\mathrm{OD}}>T_{\mathrm{m}}$ (i.e., relatively small $M_{n}$ corresponding to M08 and M11) and curve b represents the case of $T_{\mathrm{OD}} \gg T_{\mathrm{m}}$ (i.e., large $M_{n}$ corresponding to M44 and M62). The important point is that $\Delta F_{\mathrm{m}}$ meets with $\Delta F_{\mathrm{c}}$ at $T_{\mathrm{A}}$ just below $T_{\mathrm{m}}$ for curve a, and hence the microdomain structure is stable compared to the lamellar morphology only in a narrow temperature range between $T_{\mathrm{A}}$ and $T_{\mathrm{m}}$. It is expected from Figure 9 that this temperature range expands with increasing $M_{n}$ (curve a $\rightarrow$ curve b) and simultaneously the energy barrier between the homogeneous melt and the microdomain structure becomes large. Therefore, the temperature range, where the lamellar morphology is stable and hence the morphological transition occurs spontaneously, shifts gradually to the lower range with increasing $M_{n}$. The increasing energy barrier between the homogeneous melt and the microdomain structure is another factor to prevent the spontaneous morphological transition, and eventually the microdomain structure remains unchanged for the copolymers with a large $M_{n}$. Actually the kinetic factors such as the diffusion of the constituent block copolymers intervene into the morphology formation to make the transition kinetics more complicated. Figure 9 explains qualitatively the present SAXS and DSC results about the $M_{n}$ and $T_{\mathrm{c}}$ dependence of the morphology formation in PCL- $b$-PB copolymers.

\section{CONCLUSIONS}

We have investigated the morphology formed in a series of asymmetric poly ( $\varepsilon$-caprolactone)-block-polybutadiene (PCL- $b$-PB) copolymers as a function of total molecular weight $M_{n}$ and crystallization temperature $T_{\mathrm{c}}$. The PCL block is highly crystalline and all the copolymers have a cylindrical or spherical microdomain structure in the melt with the PCL block inside. The following conclusions were obtained.

1. The morphological transition (microdomain structure $\rightarrow$ lamellar morphology) was clearly observed by the crystallization of PCL blocks for the copolymers with $M_{n}$ $\leq 19000$ and the details of final morphology were significantly dependent on $T_{\mathrm{c}}$, as usually observed in the crystallization of homopolymers. The DSC curves showed a double melting behavior at a limited range of $T_{\mathrm{c}}$, suggesting the two different morphologies coexist in the system. However, the TEM observations, together with the SAXS results, showed the usual lamellar morphology instead of any coexistence of two distinctly different morphologies.

2. The microdomain structure did not change by the crystallization for PCL- $b$-PB copolymers with $M_{n} \geq$ 44000 , where the PCL crystallinity $\chi_{\mathrm{c}}$ was extremely reduced or almost zero even though the PCL block is highly crystalline by nature. The stability of the existing microdomain structure surpasses the large enthalpic penalty of the PCL block to be nearly amorphous in the cylindrical or spherical microdomains.

3. When $M_{n}=30000$, the morphological transition was clearly observed by SAXS and TEM, but the principal repeating distance of the morphology did not change, suggesting the possible epitaxial relationship between the microdomain structure and lamellar morphology for all $T_{\mathrm{c}}$ 's investigated.

Acknowledgment. This work was supported in part by Tokuyama Science Foundation. A. R. acknowledges the financial assistance from Heiwa Nakajima Foundation and Association of International Education.

\section{REFERENCES}

1. I. W. Hamley, "The Physics of Block Copolymers", Oxford University Press, Oxford 1998.

2. S. Ishikawa, K. Ishizu, and T. Fukutomi, Polym. Commun., 32, 374 (1991).

3. R. Unger, D. Beyer, and E. Donth, Polymer, 32, 3305 (1991). 
4. K. C. Douzinas and R. E. Cohen, Macromolecules, 25, 5030 (1992).

5. S. Nojima, K. Kato, S. Yamamoto, and T. Ashida, Macromolecules, 25, 2237 (1992).

6. P. Rangarajan, R. A. Register, and L. J. Fetters, Macromolecules, 26, 4640 (1993).

7. P. Kofinas and R. E. Cohen, Macromolecules, 28, 336 (1995).

8. S. Nojima, S. Yamamoto, and T. Ashida, Polym. J., 27, 673 (1995).

9. L. Liu, B. Jiang, and E. Zhou, Polymer, 37, 3937 (1996).

10. I. W. Hamley, J. P. A. Fairclough, N. J. Terrill, A. J. Ryan, P. M. Lipic, F. S. Bates, and E. T. Andrews, Macromolecules, 29 , 8835 (1996).

11. Z. Gan, B. Jiang, and J. Zhang, J. Appl. Polym. Sci., 59, 961 (1996).

12. S. Nojima, K. Hashizume, A. Rohadi, and S. Sasaki, Polymer, 38, 2711 (1997)

13. A. J. Ryan, J. P. A. Fairclough, I. W. Hamley, S. M. Mai, and C. Booth, Macromolecules, 30, 1723 (1997).

14. D. J. Quiram, R. A. Register, and G. R. Marchand, Macromolecules, 30, 4551 (1997).

15. I. W. Hamley, J. P. A. Fairclough, F. S. Bates, and A. J. Ryan, Polymer, 39, 1429 (1998).

16. S. Nojima, M. Fujimoto, H. Kakihira, and S. Sasaki, Polym. J., 30, 968 (1998).

17. D. J. Quiram, R. A. Register, G. R. Marchand, and D. H. Adamson, Macromolecules, 31, 4891 (1998).

18. G. Floudas, G. Reiter, O. Lambert, and P. Pumas, Macromolecules, 31, 7279 (1998).

19. B. Bogdanov, A. Vidts, E. Schacht, and H. Berghmans, Mac- romolecules, 32, 726 (1999).

20. V. Balsamo and R. Stadler, Macromolecules, 32, 3994 (1999).

21. E. A. DiMarzio, C. M. Guttman, and J. D. Hoffman, Macromolecules, 13, 1194 (1980).

22. M. D. Whitmore and J. Noolandi, Macromolecules, 21, 1482 (1988).

23. B. Wunderlich, "Macromolecular Physics 1-3", Academic Press, New York, N.Y., 1973-1980

24. S. Nojima, D. Wang, and T. Ashida, Polym. J., 23, 1473 (1991).

25. S. Nojima, H. Nakano, Y. Takahashi, and T. Ashida, Polymer, 35, 3479 (1994).

26. K. Ito and Y. Yamashita, Macromolecules, 11, 68 (1978).

27. V. Crescenzi, G. Manzini, G. Galzolari, and C. Borri, Eur. Polym. J., 8, 449 (1972).

28. S. Nojima, M. Kuroda, and S. Sasaki, Polym. J., 29, 642 (1997).

29. S. Nojima, H. Tanaka, A. Rohadi, and S. Sasaki, Polymer, 39, 1727 (1998).

30. R. K. Verma, V. Velikov, R. G. Kandar, H. Marand, B. Chu, and B. S. Hsiao, Polymer, 37, 5357 (1996).

31. F. J. M. Rodriguez, P. J. Phillips, and J. S. Lin, Macromolecules, 29, 7491 (1996).

32. P. Rangarajan, C. F. Haisch, R. A. Register, D. H. Adamson, and L. J. Fetters, Macromolecules, 30, 494 (1997).

33. J. D. Vavasour and M. D. Whitmore, Macromolecules, 25, 5477 (1992).

34. M. W. Matsen and M. Schick, Phys. Rev. Lett., 72, 2660 (1994). 